\title{
OUTCOME OF LAPAROSCOPIC DONOR NEPHRECTOMY: OUR INSTITUTIONAL EXPERIENCE
}

\author{
Rajaraman Thiagarajan1, Balaji A. R², Ayesha Shaheen ${ }^{3}$, Chandramurali Raveendran'4, Subhakanesh $S^{5}$, Ashok Kumar $R^{6}$, Jessima $S^{7}$
}

1 Professor and HOD, Department of Urology, Govt. Stanley Medical College.

${ }^{2}$ Assistant Professor, Department of Urology, Govt. Stanley Medical College.

${ }^{3}$ Assistant Professor, Department of Urology, Govt. Stanley Medical College.

${ }^{4}$ Post Graduate, Department of Urology, Govt. Stanley Medical College.

5 Post Graduate, Department of Urology, Govt. Stanley Medical College.

${ }^{6}$ Post Graduate, Department of Urology, Govt. Stanley Medical College.

7 Post Graduate, Department of Urology, Govt. Stanley Medical College.

\section{ABSTRACT}

Renal Transplantation has become established as the treatment of choice for patients with End Stage Renal Disease requiring renal replacement therapy. Laparoscopic Donor Nephrectomy is performed across a few centres across the country. Laparoscopic donor nephrectomy is advantageous in all aspect of donor surgery like lower operating time, less blood loss, lower post-operative pain, small surgical site scar and lesser requirement of analgesics. Though there is an increased warm ischaemia time, it has not impacted on the graft outcome. Our series also demonstrates the above benefit.

\section{KEYWORDS}

Laparoscopic Donor Nephrectomy, Post-Operative Pain, Lower Blood Loss.

HOW TO CITE THIS ARTICLE: Thiagarajan R, Balaji AR, Shaheen A, et al. Outcome of laparoscopic donor nephrectomy: our institutional experience. J. Evolution Med. Dent. Sci. 2016;5(42):2625-2630, DOI: 10.14260/jemds/2016/614

\section{INTRODUCTION}

Renal Transplantation has become established as the treatment of choice for patients with End Stage Renal Disease requiring renal replacement therapy. ${ }^{1}$ It provides the patient with a good quality of life compared to other modalities of renal replacement therapy. Compared to Deceased Donor Renal Transplantation, Living Donor Renal Transplantation is associated with better graft and patient survival, economical and logistical benefits. ${ }^{2}$ Despite this, live donor transplant has not gained wide spread acceptance among potential donors due to various factors like prolonged convalescence, postoperative pain and the resultant economic loss. Laparoscopic Donor Nephrectomy was developed in order to circumvent these shortcomings of open donor nephrectomy in the early $90 \mathrm{~s}^{3}$ Since then laparoscopic donor nephrectomy has become the standard of care in many well reputed centres across the world. ${ }^{4}$ Open Donor Nephrectomy, traditionally, has been associated with excellent results as far patient's morbidity and mortality are concerned. ${ }^{5}$ Hence, though Laparoscopic Donor Nephrectomy is an enticing procedure in terms of convalescence and shorter stay, to supplant Open Donor Nephrectomy as the procedure of choice it has to match or exceed open donor nephrectomy in terms of outcome of the donor and outcome of the recipient. 6

As far as the Indian scenario is concerned, Laparoscopic Donor Nephrectomy is performed across a few centres across the country. ${ }^{1}$ A long and steep learning curve and lack of logistical support have proven to be a major stumbling block for aspiring surgeons.

Financial or Other, Competing Interest: None.

Submission 09-04-2016, Peer Review 05-05-2016,

Acceptance 11-05-2016, Published 26-05-2016.

Corresponding Author:

Chandramurali Raveendran,

Department of Urology

NSB $6^{\text {th }}$ Floor, Govt. Stanley Medical College,

Old Jail Road, Royapuram,

Chennai-600001

Tamilnadu,

India.

E-mail: chandramuralir@gmail.com

DOI: $10.14260 /$ jemds/2016/614
In our institution, the Renal Transplantation Programme has been going on very successfully in terms of the volume and result for the past several years. Both Live and Deceased Donor Transplants have been taking place on a regular basis. Our first Laparoscopic Live Donor Transplant was done in 2013. Since then we have performed a sizeable number of laparoscopic donor transplants. In this study, we have strived to make a methodical study and comparison of the Live Open Donor Nephrectomy and Laparoscopic Donor Nephrectomy since its inception in our institution.

\section{AIMS AND OBJECTIVES}

1. To study Laparoscopic Donor Nephrectomy in our institution.

2. To compare Laparoscopic and Open Donor Nephrectomies in our institution in terms of graft and patient outcomes.

\section{MATERIALS AND METHODS}

In Government Stanley Medical College, Chennai, during the period - August 2013 - December 2015, 60 cases of donor nephrectomy were studied. The study design is prospective and observational.

\section{Inclusion Criteria}

- Donors 25 to 50 years.

- Left donor nephrectomy.

- $\quad$ Single vessel.

- ABO compatible donors.

- Donors with no associated cardiovascular and pulmonary disease.

- Absence of renal disease/infection/malignancy.

\section{Exclusion Criteria}

- Donors with hypertension and diabetics.

- Donors age $<25$ and $>50$ years.

- Donors with associated renal disease/infection/stones.

- Donors with mental dysfunction.

- Donors with transmissible malignancy.

- Anomalous kidney. 
- Donors with transmissible disease.

\section{INFERENCES}

Age Distribution among Donors

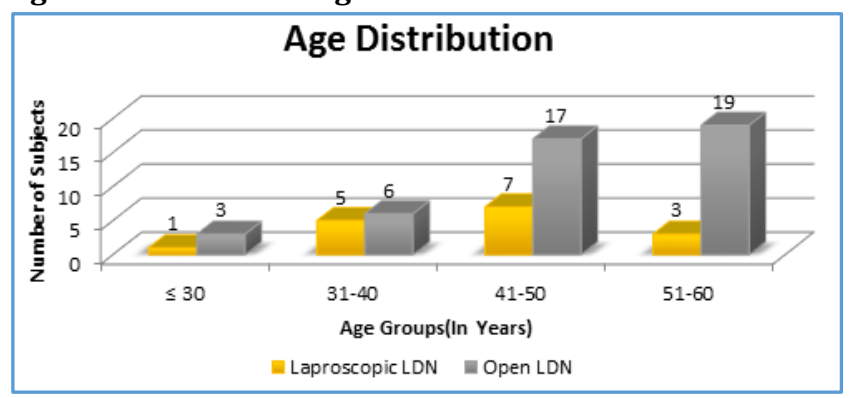

\begin{tabular}{|c|c|c|c|c|}
\hline $\begin{array}{c}\text { Age Groups } \\
\text { (In Years) }\end{array}$ & $\begin{array}{c}\text { Laparoscopic } \\
\text { LDN }\end{array}$ & $\mathbf{\%}$ & Open LDN & $\mathbf{\%}$ \\
\hline$\leq 30$ & 1 & 6.25 & 3 & 6.67 \\
\hline $31-40$ & 5 & 31.25 & 6 & 13.33 \\
\hline $41-50$ & 7 & 43.75 & 17 & 37.78 \\
\hline $51-60$ & 3 & 18.75 & 19 & 42.22 \\
\hline Total & $\mathbf{1 6}$ & $\mathbf{1 0 0}$ & $\mathbf{4 5}$ & $\mathbf{1 0 0}$ \\
\hline \multicolumn{4}{|c|}{ Table 1.1 } \\
\hline
\end{tabular}

\begin{tabular}{|c|c|c|}
\hline $\begin{array}{c}\text { Age Groups (In } \\
\text { Years) }\end{array}$ & $\begin{array}{c}\text { Laparoscopic } \\
\text { LDN }\end{array}$ & $\begin{array}{c}\text { Open } \\
\text { LDN }\end{array}$ \\
\hline $\mathrm{N}$ & 16 & 45 \\
\hline Mean & 43.50 & 47.62 \\
\hline SD & 7.10 & 8.81 \\
\hline \multicolumn{2}{|c|}{$\begin{array}{c}\text { P value } \\
\text { Unpaired t test }\end{array}$} \\
\hline \multicolumn{2}{|c|}{ Table 1.2 } \\
\hline
\end{tabular}

\section{Sex Distribution among Donors}

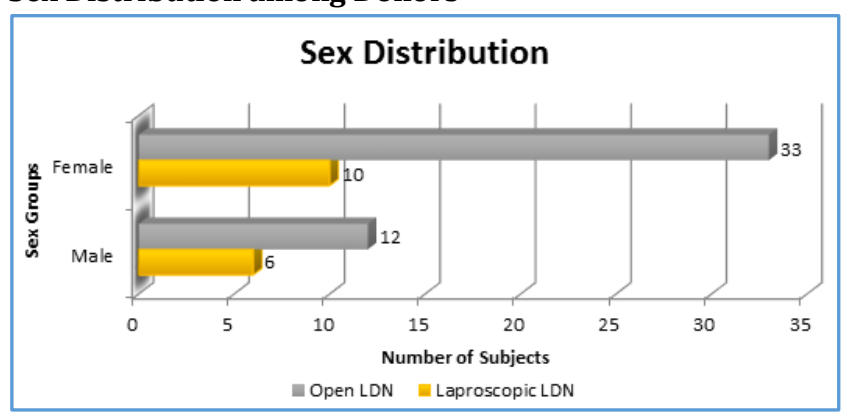

\begin{tabular}{|c|c|c|c|c|}
\hline $\begin{array}{c}\text { Sex } \\
\text { Groups }\end{array}$ & $\begin{array}{c}\text { Laparoscopic } \\
\text { LDN }\end{array}$ & $\%$ & $\begin{array}{l}\text { Open } \\
\text { LDN }\end{array}$ & $\%$ \\
\hline Male & 6 & 37.5 & 12 & 26.67 \\
\hline Female & 10 & 62.5 & 33 & 73.33 \\
\hline Total & 16 & 100 & 45 & $\begin{array}{c}100.0 \\
0\end{array}$ \\
\hline \multicolumn{3}{|c|}{ Chi Square Statistic } & \multicolumn{2}{|c|}{0.666} \\
\hline \multicolumn{3}{|c|}{ Degrees of Freedom } & \multicolumn{2}{|c|}{1} \\
\hline \multicolumn{3}{|c|}{ P value Chi Square Test } & \multicolumn{2}{|c|}{0.414} \\
\hline
\end{tabular}

Blood Group among Donors

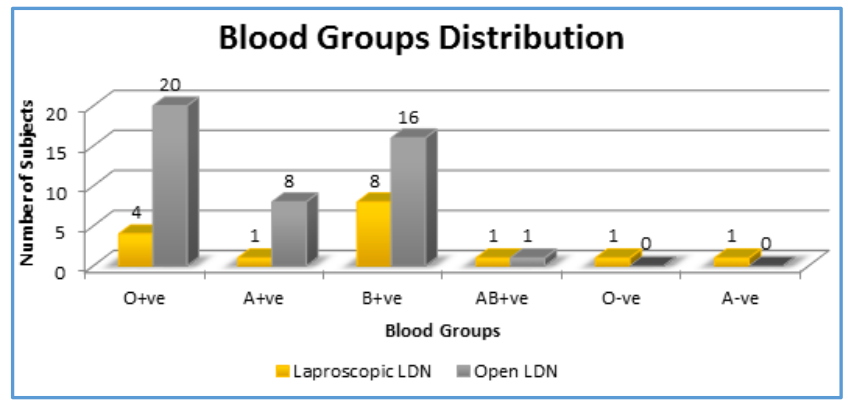

\begin{tabular}{|c|c|c|c|c|}
\hline $\begin{array}{l}\text { Blood } \\
\text { Groups } \\
\end{array}$ & $\begin{array}{c}\text { Laparoscopic } \\
\text { LDN } \\
\end{array}$ & $\%$ & $\begin{array}{c}\text { Open } \\
\text { LDN }\end{array}$ & $\%$ \\
\hline O+ve & 4 & 25 & 20 & 44.44 \\
\hline A+ve & 1 & 6.25 & 8 & 17.78 \\
\hline B+ve & 8 & 50 & 16 & 35.56 \\
\hline $\mathrm{AB}+\mathrm{ve}$ & 1 & 6.25 & 1 & 2.22 \\
\hline O-ve & 1 & 6.25 & 0 & 0.00 \\
\hline A-ve & 1 & 6.25 & 0 & 0.00 \\
\hline Total & 16 & 100 & 45 & 100.00 \\
\hline \multicolumn{3}{|c|}{ Chi Square Statistic } & \multicolumn{2}{|c|}{9.03} \\
\hline \multicolumn{3}{|c|}{ Degrees of Freedom } & \multicolumn{2}{|c|}{5} \\
\hline \multicolumn{3}{|c|}{$\mathrm{P}$ value Chi Square Test } & \multicolumn{2}{|c|}{0.108} \\
\hline \multicolumn{5}{|c|}{ Table 3} \\
\hline
\end{tabular}

Weight Distribution among Donors

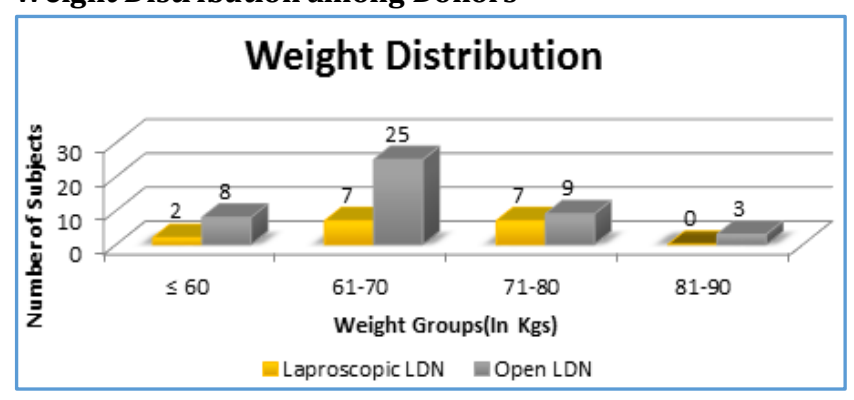

\begin{tabular}{|c|c|c|c|c|}
\hline $\begin{array}{c}\text { Weight } \\
\text { Groups } \\
\text { (In Kgs) }\end{array}$ & $\begin{array}{c}\text { Laparoscopic } \\
\text { LDN }\end{array}$ & $\mathbf{\%}$ & $\begin{array}{c}\text { Open } \\
\text { LDN }\end{array}$ & $\%$ \\
\hline$\leq 60$ & 2 & 12.5 & 8 & 17.78 \\
\hline $61-70$ & 7 & 43.75 & 25 & 55.56 \\
\hline $71-80$ & 7 & 43.75 & 9 & 20.00 \\
\hline $81-90$ & 0 & 0 & 3 & 6.67 \\
\hline Total & $\mathbf{1 6}$ & $\mathbf{1 0 0}$ & $\mathbf{4 5}$ & $\mathbf{1 0 0}$ \\
\hline \multicolumn{5}{|c|}{ Table 4.1 } \\
\hline
\end{tabular}

\begin{tabular}{|c|c|c|}
\hline $\begin{array}{c}\text { Weight Groups (In } \\
\text { Kgs) }\end{array}$ & $\begin{array}{c}\text { Laparoscopic } \\
\text { LDN }\end{array}$ & $\begin{array}{c}\text { Open } \\
\text { LDN }\end{array}$ \\
\hline N & 16 & 45 \\
\hline Mean & 69.125 & 67.28889 \\
\hline SD & 7.283543 & 7.593724 \\
\hline \multicolumn{2}{|c|}{ P value } & 0.399214 \\
\hline Unpaired t test & Table 4.2 \\
\hline \multicolumn{2}{|c|}{}
\end{tabular}


Height Distribution among Donors

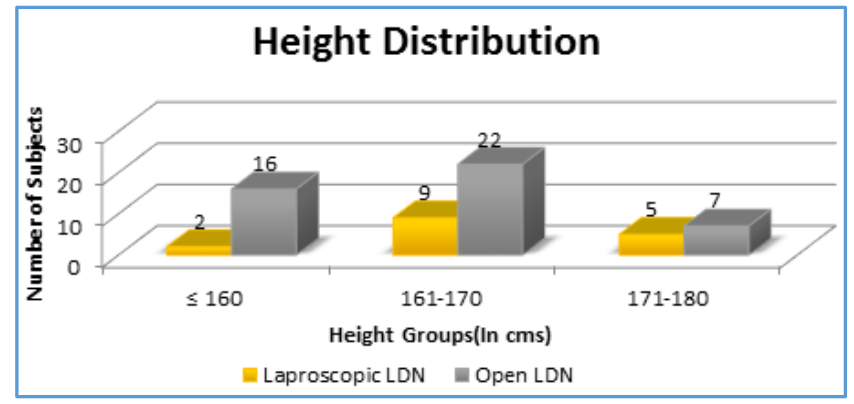

\begin{tabular}{|c|c|c|c|c|}
\hline $\begin{array}{c}\text { Height } \\
\text { Groups } \\
\text { (In cms) }\end{array}$ & $\begin{array}{c}\text { Laparoscopic } \\
\text { LDN }\end{array}$ & $\mathbf{\%}$ & $\begin{array}{c}\text { Open } \\
\text { LDN }\end{array}$ & $\mathbf{\%}$ \\
\hline$\leq 160$ & 2 & 12.5 & 16 & 35.56 \\
\hline $161-170$ & 9 & 56.25 & 22 & 48.89 \\
\hline $171-180$ & 5 & 31.25 & 7 & 15.56 \\
\hline Total & $\mathbf{1 6}$ & $\mathbf{1 0 0}$ & $\mathbf{4 5}$ & $\mathbf{1 0 0}$ \\
\hline \multicolumn{4}{|c|}{ Table 5.1 } \\
\hline
\end{tabular}

\begin{tabular}{|c|c|c|}
\hline $\begin{array}{c}\text { Height Groups } \\
\text { (In cms) }\end{array}$ & Laparoscopic LDN & Open LDN \\
\hline $\mathrm{N}$ & 16 & 45 \\
\hline Mean & 167.4375 & 163.9778 \\
\hline SD & 6.397591 & 6.264942 \\
\hline \multicolumn{2}{|c|}{ P value Unpaired t test } & 0.073097 \\
\hline
\end{tabular}

\section{Blood Urea Levels among Donors}

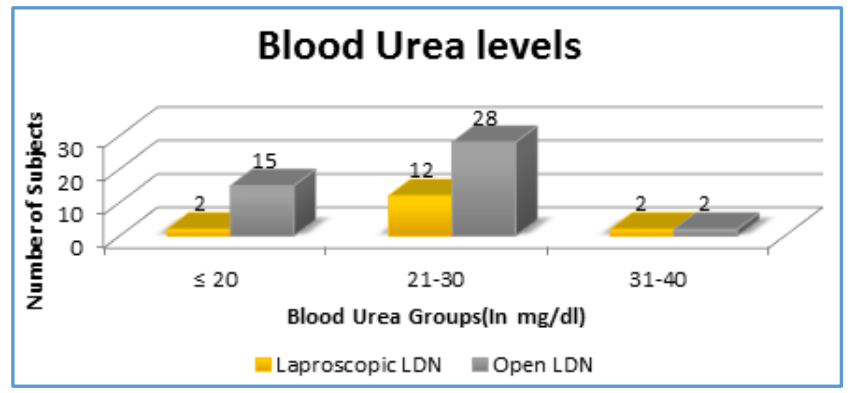

\begin{tabular}{|c|c|c|c|c|}
\hline $\begin{array}{c}\text { Blood } \\
\text { Urea } \\
\text { (In mg/dL) }\end{array}$ & $\begin{array}{c}\text { Laparoscopic } \\
\text { LDN }\end{array}$ & $\mathbf{\%}$ & $\begin{array}{c}\text { Open } \\
\text { LDN }\end{array}$ & $\mathbf{\%}$ \\
\hline$\leq 20$ & 2 & 12.5 & 15 & 33.33 \\
\hline $21-30$ & 12 & 75 & 28 & 62.22 \\
\hline $31-40$ & 2 & 12.5 & 2 & 4.44 \\
\hline Total & $\mathbf{1 6}$ & $\mathbf{1 0 0}$ & $\mathbf{4 5}$ & $\mathbf{1 0 0}$ \\
\hline \multicolumn{5}{|c|}{ Table 6.1 } \\
\hline
\end{tabular}

\begin{tabular}{|c|c|c|}
\hline $\begin{array}{c}\text { Blood Urea (In } \\
\mathrm{mg} / \mathrm{dL})\end{array}$ & $\begin{array}{c}\text { Laparoscopic } \\
\text { LDN }\end{array}$ & $\begin{array}{l}\text { Open } \\
\text { LDN }\end{array}$ \\
\hline $\mathrm{N}$ & 16 & 45 \\
\hline Mean & 26.125 & 23.02222 \\
\hline SD & 4.031129 & 4.540169 \\
\hline \multicolumn{2}{|c|}{$P$ value Unpaired t test } & $\begin{array}{c}0.015987 \\
*\end{array}$ \\
\hline & le 6.2 & \\
\hline
\end{tabular}

Serum Creatinine Levels among Donors

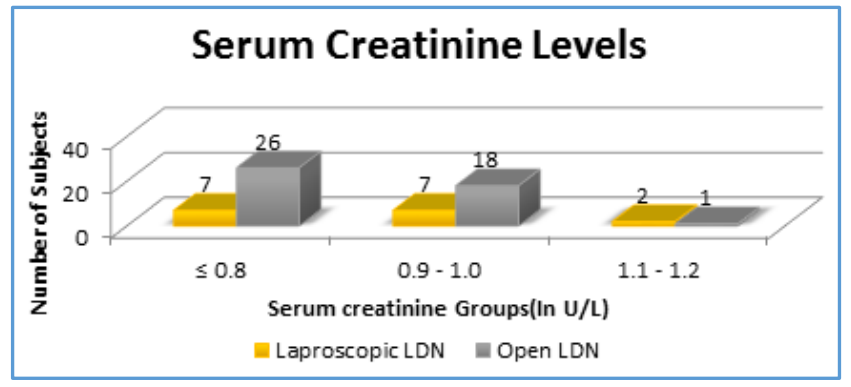

\begin{tabular}{|c|c|c|c|c|}
\hline $\begin{array}{c}\text { Serum } \\
\text { Creatinine } \\
\text { (In U/L) }\end{array}$ & $\begin{array}{c}\text { Laparoscopic } \\
\text { LDN }\end{array}$ & $\mathbf{\%}$ & $\begin{array}{c}\text { Open } \\
\text { LDN }\end{array}$ & $\mathbf{\%}$ \\
\hline$\leq 0.8$ & 7 & 43.75 & 26 & 57.78 \\
\hline $0.9-1.0$ & 7 & 43.75 & 18 & 40.00 \\
\hline $1.1-1.2$ & 2 & 12.5 & 1 & 2.22 \\
\hline Total & $\mathbf{1 6}$ & $\mathbf{1 0 0}$ & $\mathbf{4 5}$ & $\mathbf{1 0 0}$ \\
\hline \multicolumn{4}{|c|}{ Table 7.1 } \\
\hline
\end{tabular}

\begin{tabular}{|c|c|c|}
\hline $\begin{array}{c}\text { Serum Creatinine } \\
\text { (In U/L) }\end{array}$ & Laparoscopic LDN & Open LDN \\
\hline N & 16 & 45 \\
\hline Mean & 0.89375 & 0.837778 \\
\hline SD & 0.118145 & 0.113396 \\
\hline P value Unpaired t test & 0.1123 \\
\hline \multicolumn{3}{|c|}{ Table 7.2 } \\
\hline \multicolumn{2}{|c|}{} \\
\hline
\end{tabular}

Haemoglobin Levels among Donors

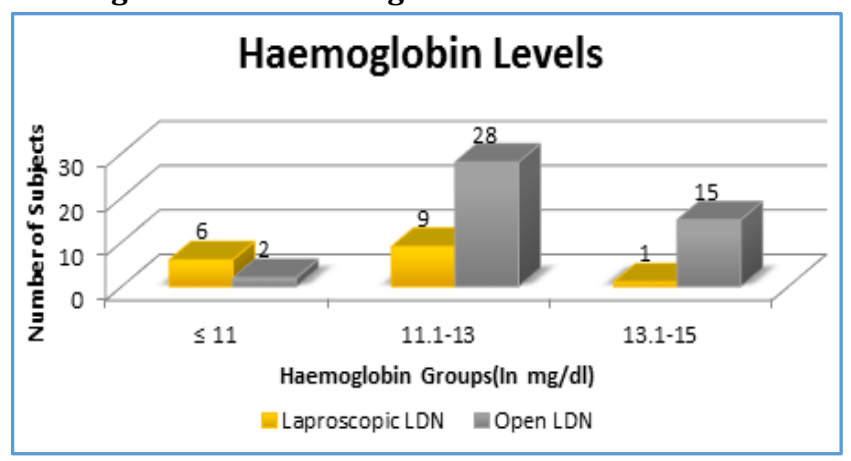

\begin{tabular}{|c|c|c|c|c|}
\hline $\begin{array}{c}\text { Haemoglob } \\
\text { in } \\
\text { (In } \\
\text { mg/dL) }\end{array}$ & $\begin{array}{c}\text { Laparoscopic } \\
\text { LDN }\end{array}$ & $\mathbf{\%}$ & $\begin{array}{c}\text { Open } \\
\text { LDN }\end{array}$ & $\%$ \\
\hline$\leq 11$ & 6 & 37.5 & 2 & 4.44 \\
\hline $11.1-13$ & 9 & 56.25 & 28 & 62.22 \\
\hline $13.1-15$ & 1 & 6.25 & 15 & 33.33 \\
\hline Total & $\mathbf{1 6}$ & $\mathbf{1 0 0}$ & $\mathbf{4 5}$ & $\mathbf{1 0 0}$ \\
\hline \multicolumn{4}{|c|}{ Table 8.1 } \\
\hline
\end{tabular}

\begin{tabular}{|c|c|c|}
\hline $\begin{array}{c}\text { Haemoglobin (In } \\
\mathbf{m g} / \mathbf{d L})\end{array}$ & $\begin{array}{c}\text { Laparoscopic } \\
\text { LDN }\end{array}$ & Open LDN \\
\hline $\mathrm{N}$ & 16 & 45 \\
\hline Mean & 11.7 & 12.68444 \\
\hline SD & 1.258571 & 0.889319 \\
\hline P value Unpaired t test & $0.009016^{*}$ \\
\hline \multicolumn{3}{|c|}{ Table 8.2 } \\
\hline
\end{tabular}




\section{DTPA-[R] Levels}

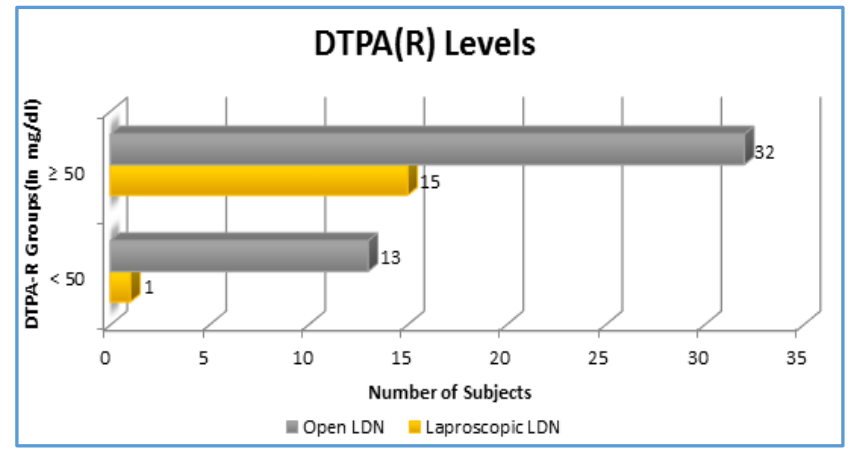

\begin{tabular}{|c|c|c|c|c|}
\hline $\begin{array}{c}\text { DTPA-R } \\
\text { (In } \\
\text { mg/dL) }\end{array}$ & $\begin{array}{c}\text { Laparoscopic } \\
\text { LDN }\end{array}$ & $\mathbf{\%}$ & $\begin{array}{c}\text { Open } \\
\text { LDN }\end{array}$ & $\%$ \\
\hline$<50$ & 1 & 6.25 & 13 & 28.89 \\
\hline$\geq 50$ & 15 & 93.75 & 32 & 71.11 \\
\hline Total & $\mathbf{1 6}$ & $\mathbf{1 0 0}$ & $\mathbf{4 5}$ & $\mathbf{1 0 0}$ \\
\hline \multicolumn{4}{|c|}{ Table 9.1 } \\
\hline
\end{tabular}

\begin{tabular}{|c|c|c|}
\hline DTPA-R (In mg/dL) & Laparoscopic LDN & Open LDN \\
\hline N & 16 & 45 \\
\hline Mean & 52.5625 & 51.37778 \\
\hline SD & 2.25 & 3.712931 \\
\hline P value Unpaired t test & 0.140425 \\
\hline \multicolumn{2}{|c|}{ Table 9.2 } \\
\hline
\end{tabular}

\section{DTPA-L}

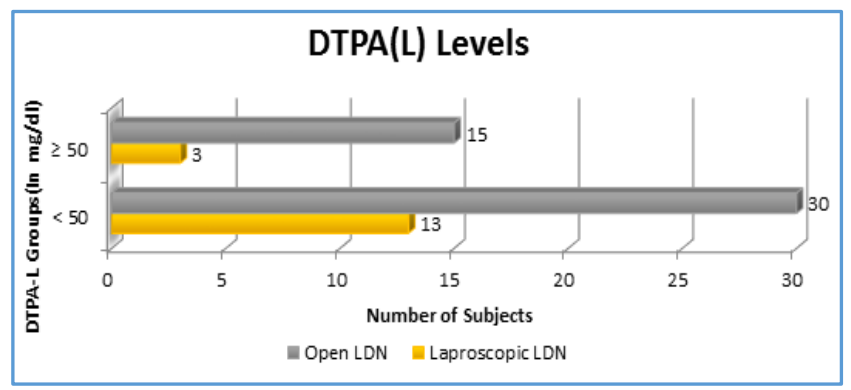

\begin{tabular}{|c|c|c|c|c|}
\hline $\begin{array}{c}\text { DTPA-L } \\
\text { (In } \\
\text { mg/dL) }\end{array}$ & $\begin{array}{c}\text { Laparoscopic } \\
\text { LDN }\end{array}$ & $\mathbf{\%}$ & $\begin{array}{c}\text { Open } \\
\text { LDN }\end{array}$ & $\%$ \\
\hline$<50$ & 13 & 81.25 & 30 & 66.67 \\
\hline$\geq 50$ & 3 & 18.75 & 15 & 33.33 \\
\hline Total & $\mathbf{1 6}$ & $\mathbf{1 0 0}$ & $\mathbf{4 5}$ & $\mathbf{1 0 0}$ \\
\hline \multicolumn{4}{|c|}{ Table 10.1 } \\
\hline
\end{tabular}

\begin{tabular}{|c|c|c|}
\hline DTPA-L (In mg/dL) & Laparoscopic LDN & Open LDN \\
\hline N & 16 & 45 \\
\hline Mean & 47.4375 & 48.62222 \\
\hline SD & 2.25 & 3.712931 \\
\hline P value Unpaired t test & 0.140425 \\
\hline \multicolumn{2}{|c|}{ Table 10.2 } \\
\hline \multicolumn{2}{|c|}{} \\
\hline \multicolumn{2}{|c|}{} \\
\hline \multicolumn{2}{|c|}{}
\end{tabular}

Comparison of Warm Ischaemia Time between Open LDN and Laparoscopic LDN

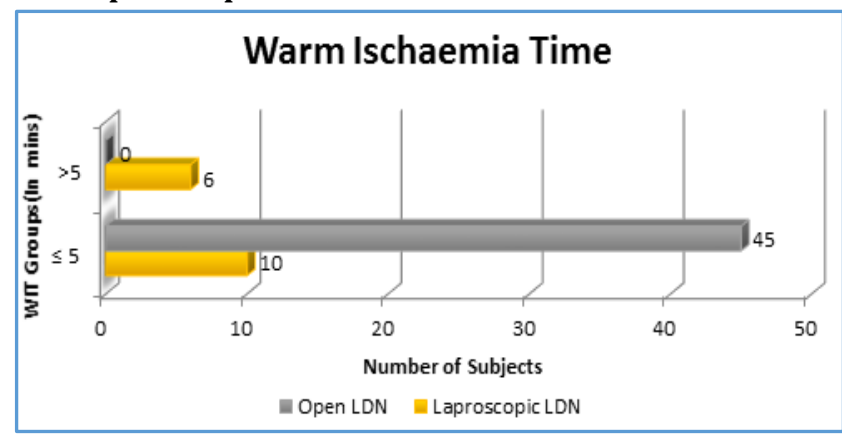

\begin{tabular}{|c|c|c|c|c|}
\hline $\begin{array}{c}\text { Warm } \\
\text { Ischaemia } \\
\text { Time } \\
\text { (In Mins) }\end{array}$ & $\begin{array}{c}\text { Laparoscopic } \\
\text { LDN }\end{array}$ & $\mathbf{\%}$ & $\begin{array}{c}\text { Open } \\
\text { LDN }\end{array}$ & $\%$ \\
\hline$\leq 5$ & 10 & 62.5 & 45 & 100.00 \\
\hline$>5$ & 6 & 37.5 & 0 & 0.00 \\
\hline Total & $\mathbf{1 6}$ & $\mathbf{1 0 0}$ & $\mathbf{4 5}$ & $\mathbf{1 0 0}$ \\
\hline \multicolumn{4}{|c}{ Table 11.1 } \\
\hline
\end{tabular}

\begin{tabular}{|c|c|c|}
\hline $\begin{array}{c}\text { Warm } \\
\text { Ischaemia Time } \\
\text { (In Mins) }\end{array}$ & $\begin{array}{c}\text { Laparoscopic } \\
\text { LDN }\end{array}$ & $\begin{array}{c}\text { Open } \\
\text { LDN }\end{array}$ \\
\hline $\mathrm{N}$ & 16 & 45 \\
\hline Mean & 5.375 & 3.288889 \\
\hline SD & 1.927866 & 0.944415 \\
\hline \multicolumn{2}{|c|}{ P value Unpaired t test } & $0.000619^{*}$ \\
\hline \multicolumn{2}{|c|}{ Table 11.2 }
\end{tabular}

\section{Graft Side}

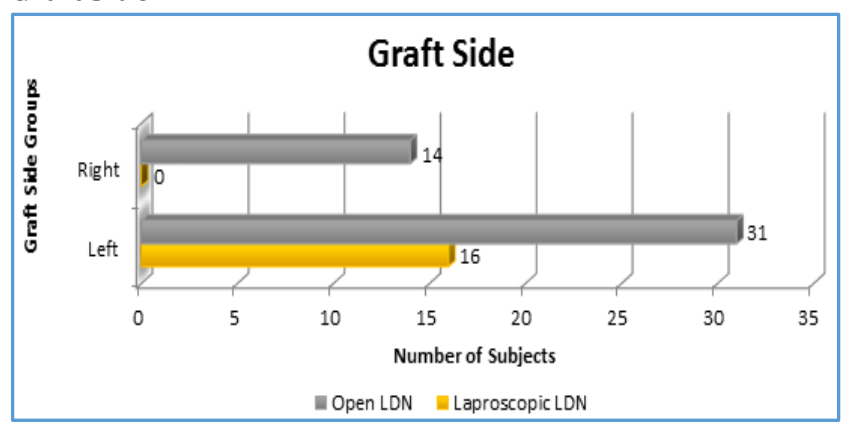

\begin{tabular}{|c|c|c|c|c|}
\hline $\begin{array}{c}\text { Graft } \\
\text { Side }\end{array}$ & $\begin{array}{c}\text { Laparoscopic } \\
\text { LDN }\end{array}$ & $\mathbf{\%}$ & $\begin{array}{c}\text { Open } \\
\text { LDN }\end{array}$ & $\%$ \\
\hline Left & 16 & 100 & 31 & 68.89 \\
\hline Right & 0 & 0 & 14 & 31.11 \\
\hline Total & $\mathbf{1 6}$ & $\mathbf{1 0 0}$ & $\mathbf{4 5}$ & $\mathbf{1 0 0}$ \\
\hline \multicolumn{3}{|c|}{ Chi Square Statistic } & 6.46 & \multicolumn{2}{|c|}{1} \\
\hline \multicolumn{3}{|c|}{ Degrees of Freedom } & Table 12 \\
\hline \multicolumn{2}{|c|}{ P value Chi Square Test } \\
\hline \multicolumn{4}{c}{ Table } \\
\hline
\end{tabular}


CT Angiography (Artery)

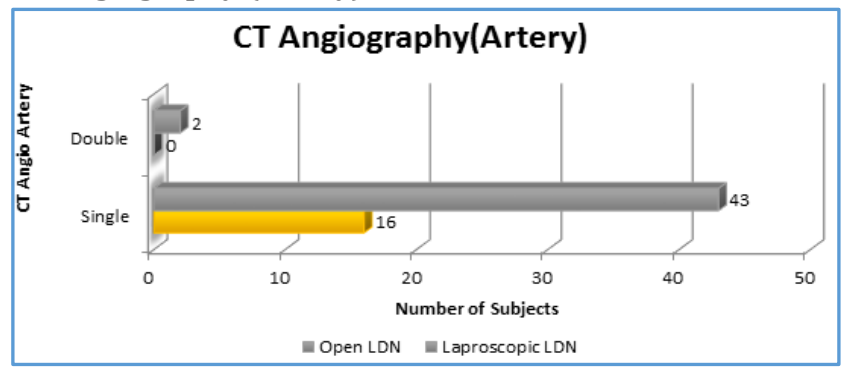

\begin{tabular}{|c|c|c|c|c|}
\hline $\begin{array}{c}\text { CT } \\
\text { Angiography } \\
\text { (Artery) }\end{array}$ & $\begin{array}{c}\text { Laparoscopi } \\
\text { c LDN }\end{array}$ & $\mathbf{\%}$ & $\begin{array}{c}\text { Open } \\
\text { LDN }\end{array}$ & $\mathbf{\%}$ \\
\hline Single & 16 & 100 & 43 & 95.56 \\
\hline Double & 0 & 0 & 2 & 4.44 \\
\hline Total & $\mathbf{1 6}$ & $\mathbf{1 0 0}$ & $\mathbf{4 5}$ & $\mathbf{1 0 0}$ \\
\hline \multicolumn{2}{|c|}{ Chi Square Statistic } & \multicolumn{2}{|c|}{0.735} \\
\hline \multicolumn{3}{|c|}{ Degrees of Freedom } & \multicolumn{2}{|c|}{0.391} \\
\hline \multicolumn{3}{|c|}{ Table 13 value Chi Square Test } \\
\hline \multicolumn{3}{|c|}{} \\
\hline
\end{tabular}

Duration of Hospital Stay

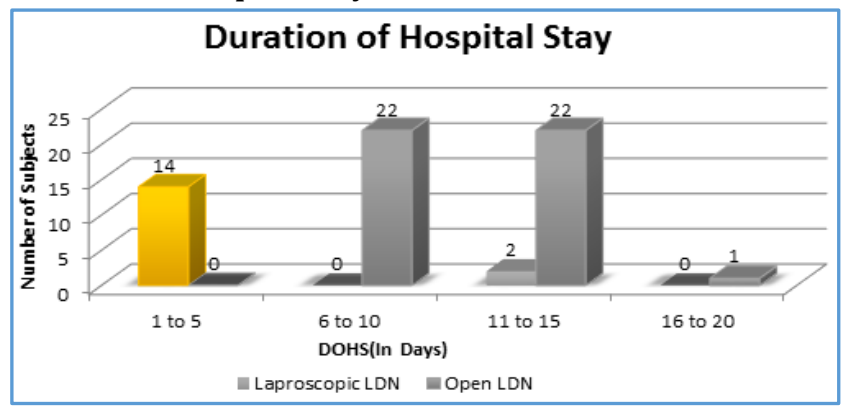

\begin{tabular}{|c|c|c|c|c|}
\hline $\begin{array}{c}\text { Duration } \\
\text { of Hospital } \\
\text { Stay } \\
\text { (In Days 0) }\end{array}$ & $\begin{array}{c}\text { Laparoscopic } \\
\text { LDN }\end{array}$ & $\mathbf{\%}$ & $\begin{array}{c}\text { Open } \\
\text { LDN }\end{array}$ & $\mathbf{\%}$ \\
\hline 1 to 5 & 14 & 87.5 & 0 & 0.00 \\
\hline 6 to 10 & 0 & 0 & 22 & 48.89 \\
\hline 11 to 15 & 2 & 12.5 & 22 & 48.89 \\
\hline 16 to 20 & 0 & 0 & 1 & 2.22 \\
\hline Total & $\mathbf{1 6}$ & $\mathbf{1 0 0}$ & $\mathbf{4 5}$ & $\mathbf{1 0 0}$ \\
\hline \multicolumn{4}{|c|}{ Table 14.1 } \\
\hline
\end{tabular}

\begin{tabular}{|c|c|c|}
\hline $\begin{array}{c}\text { Duration of Hospital } \\
\text { Stay }\end{array}$ & $\begin{array}{c}\text { Laparoscopic } \\
\text { LDN }\end{array}$ & $\begin{array}{c}\text { Open } \\
\text { LDN }\end{array}$ \\
\hline N & 16 & 45 \\
\hline Mean & 5.1875 & 10.84444 \\
\hline SD & 2.53558 & 1.609002 \\
\hline P value Unpaired t test & $0.00001^{*}$ \\
\hline \multicolumn{2}{|c|}{ Table 14.2 } \\
\hline
\end{tabular}

\section{Analgesic Requirement}

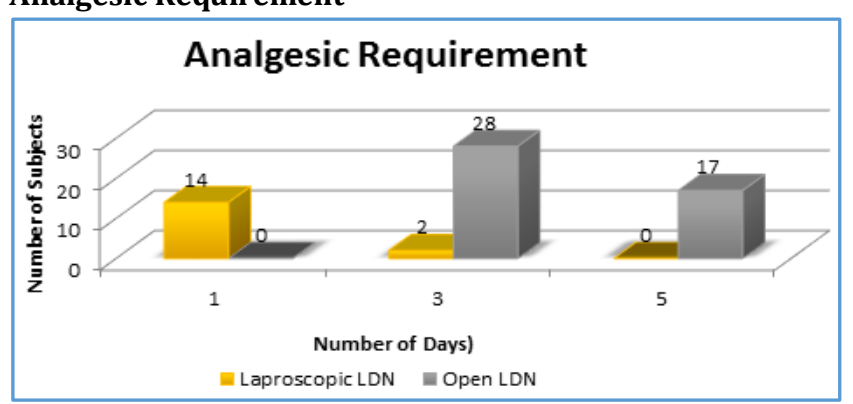

\begin{tabular}{|c|c|c|c|c|}
\hline $\begin{array}{c}\text { Analgesic } \\
\text { Requirement }\end{array}$ & $\begin{array}{c}\text { Laparoscopic } \\
\text { LDN }\end{array}$ & \% & $\begin{array}{c}\text { Open } \\
\text { LDN }\end{array}$ & \% \\
\hline 1 & 14 & 87.5 & 0 & 0.00 \\
\hline 3 & 2 & 12.5 & 28 & 62.22 \\
\hline 5 & 0 & 0 & 17 & 37.78 \\
\hline Total & $\mathbf{1 6}$ & $\mathbf{1 0 0}$ & $\mathbf{4 5}$ & $\mathbf{1 0 0}$ \\
\hline \multicolumn{5}{|c|}{ Table 15.1 } \\
\hline
\end{tabular}

\begin{tabular}{|c|c|c|}
\hline $\begin{array}{c}\text { Analgesic } \\
\text { Requirement }\end{array}$ & $\begin{array}{c}\text { Laparoscopic } \\
\text { LDN }\end{array}$ & $\begin{array}{c}\text { Open } \\
\text { LDN }\end{array}$ \\
\hline $\mathrm{N}$ & 16 & 45 \\
\hline Mean & 1.25 & 3.755556 \\
\hline SD & 0.68313 & 0.98062 \\
\hline P value Unpaired t test & $0.00001^{*}$ \\
\hline \multicolumn{2}{|c}{ Table 15.2 } \\
\hline
\end{tabular}

Operative Time

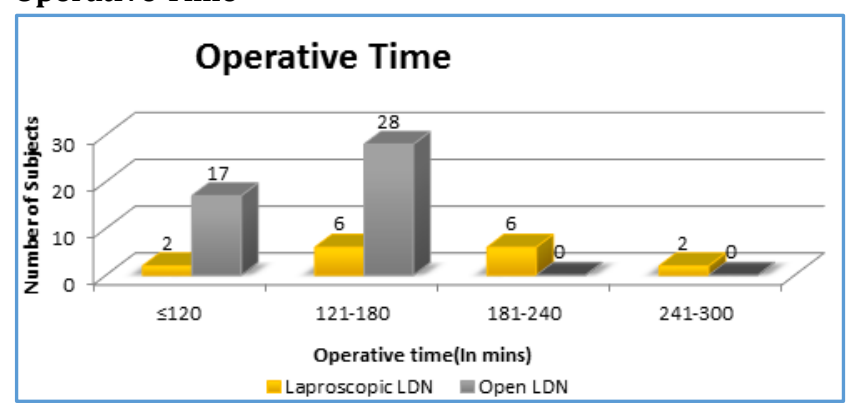

\begin{tabular}{|c|c|c|c|c|}
\hline $\begin{array}{c}\text { Operative } \\
\text { Time } \\
\text { (In Mins) }\end{array}$ & $\begin{array}{c}\text { Laparoscopic } \\
\text { LDN }\end{array}$ & $\mathbf{\%}$ & $\begin{array}{c}\text { Open } \\
\text { LDN }\end{array}$ & $\mathbf{\%}$ \\
\hline$\leq 120$ & 2 & 12.5 & 17 & 37.78 \\
\hline $121-180$ & 6 & 37.5 & 28 & 62.22 \\
\hline $181-240$ & 6 & 37.5 & 0 & 0.00 \\
\hline $241-300$ & 2 & 12.5 & 0 & 0.00 \\
\hline Total & $\mathbf{1 6}$ & $\mathbf{1 0 0}$ & $\mathbf{4 5}$ & $\mathbf{1 0 0}$ \\
\hline \multicolumn{4}{|c|}{ Table 16.1 } \\
\hline
\end{tabular}

\begin{tabular}{|c|c|c|}
\hline $\begin{array}{c}\text { Operative Time } \\
\text { (In Mins) }\end{array}$ & $\begin{array}{c}\text { Laparoscopic } \\
\text { LDN }\end{array}$ & $\begin{array}{c}\text { Open } \\
\text { LDN }\end{array}$ \\
\hline $\mathrm{N}$ & 16 & 45 \\
\hline Mean & 179.9375 & 129.8889 \\
\hline SD & 47.61578 & 24.06168 \\
\hline P value Unpaired t test & $0.00081^{*}$ \\
\hline \multicolumn{2}{|c|}{ Table 16.2 } \\
\hline
\end{tabular}

\section{Blood Loss}

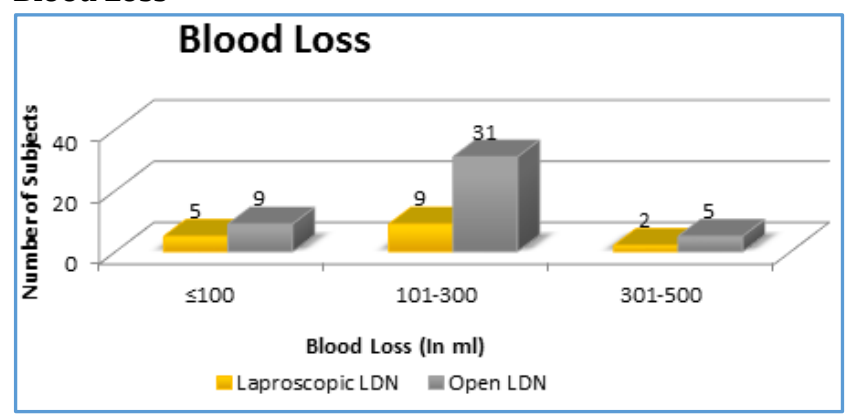




\begin{tabular}{|c|c|c|c|c|}
\hline $\begin{array}{c}\text { Blood } \\
\text { Loss } \\
\text { (In MI) }\end{array}$ & $\begin{array}{c}\text { Laparoscopic } \\
\text { LDN }\end{array}$ & $\mathbf{\%}$ & $\begin{array}{c}\text { Open } \\
\text { LDN }\end{array}$ & $\%$ \\
\hline$\leq 100$ & 5 & 31.25 & 9 & 20.00 \\
\hline $101-300$ & 9 & 56.25 & 31 & 68.89 \\
\hline $301-500$ & 2 & 12.5 & 5 & 11.11 \\
\hline Total & $\mathbf{1 6}$ & $\mathbf{1 0 0}$ & $\mathbf{4 5}$ & $\mathbf{1 0 0}$ \\
\hline \multicolumn{4}{|c|}{ Table 17.1 } \\
\hline
\end{tabular}

\begin{tabular}{|c|c|c|}
\hline Blood Loss ( In mL) & Laparoscopic LDN & Open LDN \\
\hline N & 16 & 45 \\
\hline Mean & 163.4375 & 215.5556 \\
\hline SD & 93.37592 & 119.5742 \\
\hline P value Unpaired t test & 0.08503 \\
\hline \multicolumn{2}{|c|}{ Table 17.2 } \\
\hline
\end{tabular}

\section{DISCUSSION}

Our study is a prospective study and we have evaluated 16 laparoscopic donor nephrectomy and 45 open donor nephrectomy. We have compared our study with similar studies, where multiple factors were analysed for outcome of donors following laparoscopy and the graft function in recipients. 1,6,7,8,9 In our series, we have selected only a left side kidney for laparoscopic procedure which is easier technically because of long renal vein on left side. This has been already reported in many studies including hands. ${ }^{9,10}$

All the 16 kidneys harvested by laparoscopic method were transplanted successfully in the recipients with only $2(12 \%)$ conversion to open due to bleeding from posterior lumbar vein where bleeding was controlled, but we found difficulty in repairing it laparoscopically. Conversion rate in SGPGI is $10.1 \% .{ }^{1}$ Mean operating time was $179.9 \pm 47.6$ minutes, which were comparable with other studies of about 225 minutes on an average.1,10,11,12 Our operative time has been lesser in duration with all the above mentioned studies.

Blood loss in our study was $169 \pm 93 \mathrm{~mL}$, which is comparable with other studies.1,10,11 Most series have shown decreased blood loss in LDN on comparing with open surgery. The analgesic requirement of LDN in our series was 1.25 days, which was significantly lower when compared to open group (mean 3.75 days) P value 0.00001. Similar studies also shown decreased analgesic requirement in LDN.1,10,11,13

Our study shows duration of hospital study was lower in LDN (Mean 5.1 days) as compared to open group (Mean 10.8 days), statistically significant p-value 0.00001 , as it was seen in other series.1,6,10,11 We monitored serial values of serum creatinine and urine output of the recipients postoperatively, which are good indicators of renal graft function. Many studies showed that though there was prolonged warm ischaemia, there was no significant difference seen in initial graft function and more so the final outcome.1,6.9.10,11 This has been seconded by our study, which showed prolonged warm ischaemia time seen in laparoscopic donor nephrectomy (5.3 minutes) with initial graft function similar to that of open surgery. Limitations of our study is small number of cases. Only leftsided laparoscopic donor nephrectomy has been done. We have not done donor nephrectomy with any added risk factors. A larger randomized controlled study is required to confirm the finding of our study and to establish the merits of laparoscopic donor nephrectomy.

\section{CONCLUSION}

Laparoscopic donor nephrectomy is an effective, safe and rewarding procedure. Although, it is time consuming and technically demanding with steep learning curve, once acquired has produced results as comparable and also better in some aspects than open surgeries. The analgesic requirement, duration of hospital stay and the blood loss were less with the laparoscopic surgery than open surgery. Results of graft functioning of laparoscopically harvested kidneys were equivalent to those kidneys harvested from open surgery. All these show that laparoscopic donor nephrectomy can be made as the procedure of choice in future.

\section{REFERENCES}

1. Arvind NK, Kumar A. Laparoscopic live donor nephrectomy: an Indian perspective. Indian J Urol 2002;19(1):29-37.

2. Nogueira JM, Cangro CB, Fink JC, et al. A comparison of recipient renal outcomes with laparoscopic versus open live donor nephrectomy. Transplantation 1999;67(5):722-8.

3. Tooher RL, Rao MM, Scott DF, et al. A systematic review of laparoscopic live-donor nephrectomy. Transplantation 2004;78(3):404-14.

4. Buell J, Edye M, Johnson L, et al. Are concerns over right laparoscopic donor nephrectomy unwarranted? Ann Surg 2001;233(5):645-51.

5. Greco F, Hoda MR, Alcaraz A, et al. Laparoscopic living donor nephrectomy: analysis of the existing literature. Eur Urol 2010;58(4):498-509.

6. Sundaram CP, Martin GL, Guise A, et al. Complications after a 5-year experience with laparoscopic donor nephrectomy: the Indiana university experience. Surg Endosc 2007;21(5):724-8.

7. Harper JD, Breda JT, Leppert JL, et al. Experience with 750 consecutive laparoscopic donor nephrectomy - is it time to use a standard classification of complications? J Urol 2010;183:1941-6.

8. Alston C, Spaliviero M, Gill IS. Laparoscopic donor nephrectomy. Urology 2005;65(5):833-9.

9. Bargman V, Sundaram CP, Bernie J, et al. Randomized trial laparoscopic donor nephrectomy with and without hand assistance. J Endourol 2006;20(10):717-22.

10. Øyen O, Andersen M, Mathisen L, et al. Laparoscopic versus open living donor nephrectomy: experience from a prospective, randomised, single centre study focusing on donor safety. Transplantation 2005;79(9):1236-40.

11. Ratner LE, Kavoussi LR, Schulam PG, et al. Comparison of laparoscopic live donor nephrectomy versus the standard open approach. Transplant Proc 1997;29112):138-9.

12. Simforoosh N, Basiri A, Tabibi A, et al. Comparison of laparoscopic and open donor nephrectomy: a randomized controlled trial. BJU Int 2005;95(6):851-5.

13. Ratner LE, Montgomery RA, Kavoussi LR. Laparoscopic live donor nephrectomy: the four-year john hopkins university experience. Nephrol Dial Transplant 1999;14(9):2090-3. 